\title{
Umbilical Cord Diameter
}

National Cancer Institute

\section{Source}

National Cancer Institute. Umbilical Cord Diameter. NCI Thesaurus. Code C117357.

The longest measured width of the umbilical cord. 